\title{
PERIODIC SOLUTIONS OF A NEURAL NETWORK MODEL WITH DELAY
}

\section{YE SUN and CHUNRUI ZHANG}

Department of Mathematics

Northeast Forestry University

Harbin 150040

P. R. China

e-mail:616292301@qq.com

math@nefu.edu.cn

\begin{abstract}
In this paper, we analyze a neural network system with delay. Firstly, by considering time delay as bifurcation parameter, we discuss the stability of the model and the conditions when Hopf bifurcation occurs. Finally, after complicated calculations with Lyapunov-Schmidt reduction, we obtain the approximate analytic expression of periodic solutions near the Hopf bifurcation points.
\end{abstract}

\section{Introduction}

The occuring of Hopf bifurcation is an important property of dynamical systems with delay, and it refers to a phenomenon that the systems have periodic solutions near the singularity when the stability of the singularity changes. After determining that a system exists Hopf

2010 Mathematics Subject Classification: 34A47, 34C23, 37C75.

Keywords and phrases: neural network, delay Hopf bifurcation, periodic solutions, LyapunovSchmidt reduction.

This research was supported by the Natural Science Foundations of Heilongjiang Province (A2015016).

Received November 23, 2016

(C) 2016 Scientific Advances Publishers 
bifurcation, under normal conditions, to determine the stability and direction of the periodic solutions [1-5], the delay differential equations need to be expressed as ordinary differential equations in abstract spaces. The system must has a 2 -dimensional center manifold which is tangent to the central subspace near the constant solutions. Hence, we only need to considering the limitation of the original system on the center manifold, actually turning it into a 2-dimensional differential equation. As long as we study the stability and the direction of periodic solutions of the ordinary differential equation, we can obtain the properties related to the original system. For example, Faria and Magalháes [6] and Liu et al. [7] all use this technique. Lyapunov-Schmidt reduction is an important method to investigate the bifurcation problems of ordinary differential equations [8]. Inspired by Yang and Guo [9], we investigate the periodic solutions of a neural network model with delay by using LyapunovSchmidt reduction [10-13] and obtain obvious expressions depending on the parameters of the original system. This method avoids numerous calculations of center manifold and normal form.

The model to study in this paper is

$$
\dot{x}=-a x(t)+b x(t-\tau)+\frac{1}{6} x(t)^{3},
$$

where the dot denotes differentiation with respect to time, and $a>0, b$ are parameters.

Our paper is organized as follows: In Section 2, we discuss the stability of the model and the conditions when Hopf bifurcation occurs. In Section 3, we obtain the approximate analytic expression of periodic solutions near the Hopf bifurcation points with Lyapunov-Schmidt reduction.

\section{Stability Analysis}

From system (1.1), we can see that there exists an zero equilibrium and the characteristic equation of the linearized system at zero solution is

$$
\lambda+a-b e^{(-\lambda \tau)}=0
$$


when $\tau=0$, it is obvious that $b<a$, the root of the equation has always negative real parts. If $b<-a$, Equation (2.1) has one positive root $\omega=\sqrt{b^{2}-a^{2}}$, $\tau_{n}=\frac{1}{\omega}\left(\pi-\arcsin \left(\frac{\omega}{-b}\right)\right)+\frac{2 n \pi}{\omega}, n=0,1,2, \cdots, \operatorname{sign}\left\{\operatorname{Re}\left(\frac{d \lambda}{d \tau}\right)^{-1}\right\}_{\tau=\tau_{n}}$ $=\frac{a}{b^{2}}>0$

Theorem 2.1. If $b<-a$, then the zero solution of system (1.1) is asymptotically stable for $\tau \in\left[0, \tau_{0}\right)$, and unstable when $\tau>\tau_{0}$. Furthermore, system (1.1) undergoes a Hopf bifurcation at the origin when $\tau=\tau_{n}(n=0,1, \cdots)$.

\section{Lyapunov-Schmidt Reduction Process}

In this section, near the Hopf bifurcation, we obtain the approximate analytic periodic solutions bifurcated from the trivial solution of the neural network by Lyapunov-Schmidt reduction method. Now, we will mainly discuss how to get the periodic solutions near the Hopf bifurcation point $\tau_{0}$.

First of all, by the transformation $\hat{s}=\frac{t}{\tau}, u(\hat{s})=x(\tau \hat{s}), \tau=\tau_{0}+\varepsilon$, $s=(1+k) \hat{s}$, then system (1.1) is equivalent to

$$
F(u, \varepsilon, k)=(1+k) \frac{d u}{d s}+\left(\tau_{0}+\varepsilon\right)\left[a u(s)-b u(s-(1+k))-\frac{1}{6} u(s)^{3}\right] .
$$

Let

$$
\begin{aligned}
& C_{p}^{0}\left[0, \frac{2 \pi}{n}\right]=u \in C^{0}(-\infty,+\infty) \mid u(s)=u\left(s+\frac{2 \pi}{n}\right), \\
& C_{p}^{1}\left[0, \frac{2 \pi}{n}\right]=u \in C^{1}(-\infty,+\infty) \mid u(s)=u\left(s+\frac{2 \pi}{n}\right) .
\end{aligned}
$$


Then $F(u, \varepsilon, k): C_{p}^{1}\left[0, \frac{2 \pi}{n}\right] \times R \times R \rightarrow C_{p}^{0}\left[0, \frac{2 \pi}{n}\right]$, the linearized equation of Equation (3.1) at zero solution is

$$
L_{0} v=F_{u}^{-}(0,0,0) v=\frac{d v}{d s}+\tau_{0} a v-\tau_{0} b v(s-1)=0,
$$

the adjoint equation is

$$
L_{0}^{*} v=F_{u}^{+}(0,0,0) v=-\frac{d v}{d s}+\tau_{0} a v-\tau_{0} b v(s+1)=0,
$$

obviously

$$
N\left(L_{0}\right)=\operatorname{span}\{\sin n s, \cos n s\}, \quad N\left(L_{0}^{*}\right)=\operatorname{span}\{\sin n s, \cos n s\} .
$$

We have the following decomposition:

$$
C_{p}^{1}\left[0, \frac{2 \pi}{n}\right]=N\left(L_{0}\right) \oplus M, \quad C_{p}^{0}\left[0, \frac{2 \pi}{n}\right]=N\left(L_{0}^{*}\right) \oplus R\left(L_{0}\right) .
$$

Define

$$
\begin{gathered}
Q: C_{p}^{0}\left[0, \frac{2 \pi}{n}\right] \rightarrow N\left(L_{0}^{*}\right), P: C_{p}^{0}\left[0, \frac{2 \pi}{n}\right] \rightarrow R\left(L_{0}\right), \\
Q v=<v, \sin n s>\sin n s+<v, \cos n s>\cos n s, P v=v-Q v,
\end{gathered}
$$

define an inner product is given by

$$
<v_{1}, v_{2}>=\frac{n}{\pi} \int_{0}^{\frac{2 \pi}{n}} v_{1} v_{2} d s
$$

Let

$$
u(s)=x \sin n s+y \cos n s+\omega(s)
$$

where $\omega(s) \in M$. By respectively projecting system (3.1) onto $R\left(L_{0}\right)$ and $N\left(L_{0}^{*}\right)$, we obtain

$$
\begin{aligned}
& P F(x \sin n s+y \cos n s+\omega(s), \varepsilon, k)=0, \\
& Q F(x \sin n s+y \cos n s+\omega(s), \varepsilon, k)=0 .
\end{aligned}
$$


Rewrite (3.4) as

$$
\begin{aligned}
\widetilde{F}(\omega ; x, y, \varepsilon, k)= & P\left[(1+k)\left(n x \cos n s-n y \sin n s+\frac{d \omega(s)}{d s}\right)\right. \\
& +a\left(\tau_{0}+\varepsilon\right)(x \sin n s+y \cos n s+\omega(s)) \\
& -b\left(\tau_{0}+\varepsilon\right)(x \sin n(s-(1+k))+y \cos n(s-(1+k))) \\
& \left.+\omega(s-(1+k))-\frac{1}{6}\left(\tau_{0}+\varepsilon\right)(x \sin n s+y \cos n s+\omega(s))^{3}\right]=0 .
\end{aligned}
$$

We know $\widetilde{F}_{\omega}(0 ; 0,0,0,0)=P L_{0}=L_{0}$, and $L_{0}$ is regular on $M$, by the implicit function theorem, it is easy to solve from system (3.6) that

$$
\omega(s)=\omega(s ; x, y, \varepsilon, k) .
$$

Obviously, $\omega(s ; 0,0,0,0) \equiv 0$, plug the system (3.7) into the system (3.5), we can obtain the equivalent bifurcation equations are

$g(x, y, \varepsilon, k)=<F(x \sin n s+y \cos n s+\omega(s ; x, y, \varepsilon, k)), \sin n s>=0, \quad(3.8)$

$h(x, y, \varepsilon, k)=<F(x \sin n s+y \cos n s+\omega(s ; x, y, \varepsilon, k)), \cos n s>=0$.

Secondly, to obtain the further information respect to the periodic solutions, we need to calculate the approximate analytic expressions of system (3.7), (3.8), and (3.9).

For the convenience of narrative, we make some notes

$$
\begin{gathered}
\omega^{0}(s)=\omega(s ; 0,0,0,0) \equiv 0, \omega_{x}^{0}(s)=\omega_{x}(s ; 0,0,0,0), \omega_{y}^{0}(s)=\omega_{y}(s ; 0,0,0,0), \\
\omega_{\varepsilon}^{0}(s)=\omega_{\varepsilon}(s ; 0,0,0,0), \omega_{k}^{0}(s)=\omega_{k}(s ; 0,0,0,0), g_{x}^{0}(s)=g_{x}(s ; 0,0,0,0), \\
h_{x}^{0}(s)=h_{x}(s ; 0,0,0,0), g_{y}^{0}(s)=g_{y}(s ; 0,0,0,0), h_{y}^{0}(s)=h_{y}(s ; 0,0,0,0), \\
g_{\varepsilon}^{0}(s)=g_{\varepsilon}(s ; 0,0,0,0), h_{\varepsilon}^{0}(s)=h_{\varepsilon}(s ; 0,0,0,0), g_{k}^{0}(s)=g_{k}(s ; 0,0,0,0), \\
h_{k}^{0}(s)=h_{k}(s ; 0,0,0,0) \cdots .
\end{gathered}
$$


Take the derivative of system (3.6) with respect to $x$, we have

$$
\frac{d \omega_{x}^{0}(s)}{d s}+a \tau_{0} \omega_{x}^{0}(s)-b \tau_{0} \omega_{x}^{0}(s-1)=0,
$$

and

$$
<\omega_{x}^{0}(s), \sin n s>=0,<\omega_{x}^{0}(s), \cos n s>=0 .
$$

We can solve that

$$
\omega_{x}^{0}(s) \equiv 0
$$

Analogously, we can obtain

$$
\begin{gathered}
\omega_{y}^{0}(s)=0, \omega_{\varepsilon}^{0}(s)=0, \omega_{k}^{0}(s)=0, \omega_{x x}^{0}(s)=0, \omega_{y y}^{0}(s)=0, \omega_{x y}^{0}(s)=0, \\
\omega_{x \varepsilon}^{0}(s)=0, \omega_{x k}^{0}(s)=0, \omega_{y \varepsilon}^{0}(s)=0, \omega_{y k}^{0}(s)=0, \omega_{\varepsilon \varepsilon}^{0}(s)=0, \omega_{k k}^{0}(s)=0 .
\end{gathered}
$$

Take three derivatives of system (3.6) with respect to $x$, we have

$$
\begin{gathered}
\frac{d \omega_{x x x}^{0}(s)}{d s}+a \tau_{0} \omega_{x x x}^{0}(s)-b \tau_{0} \omega_{x x x}^{0}(s-1)-\tau_{0} P\left[(\sin n s)^{3}\right]=0, \\
P\left[(\sin n s)^{3}\right]=-\frac{1}{4} \sin 3 n s,
\end{gathered}
$$

let

$$
\omega_{x x x}^{0}(s)=c_{1} \sin 3 n s+c_{2} \cos 3 n s+c_{3},
$$

plug the system (3.11) into the system (3.10), we can calculate that

$$
\begin{gathered}
c_{1}=\frac{-\frac{1}{4} \tau_{0}\left(a \tau_{0}-b \tau_{0} \cos 3 n\right)}{\left(a \tau_{0}-b \tau_{0} \cos 3 n\right)^{2}+\left(3 n+b \tau_{0} \sin 3 n\right)^{2}}, \\
c_{2}=\frac{\frac{1}{4} \tau_{0}\left(3 n+b \tau_{0} \sin 3 n\right)}{\left(a \tau_{0}-b \tau_{0} \cos 3 n\right)^{2}+\left(3 n+b \tau_{0} \sin 3 n\right)^{2}}, c_{3}=0 .
\end{gathered}
$$


In the same way, we can obtain

$$
\begin{aligned}
& \omega_{y y y}^{0}(s)=c_{2} \sin 3 n s-c_{1} \cos 3 n s, \omega_{x x y}^{0}(s)=-c_{2} \sin 3 n s+c_{1} \cos 3 n s, \\
& \omega_{x y y}^{0}(s)=-c_{1} \sin 3 n s-c_{2} \cos 3 n s, \omega_{x x \varepsilon}^{0}(s)=0, \omega_{x x k}^{0}(s)=0, \\
& \omega_{y y \varepsilon}^{0}(s)=0, \omega_{y y k}^{0}(s)=0, \omega_{x y \varepsilon}^{0}(s)=0, \omega_{x y k}^{0}(s)=0 .
\end{aligned}
$$

We can calculate that

$$
g_{x}^{0}(s)=<F_{x}(\omega ; x, y, \varepsilon, k), \sin n s>=a \tau_{0}-b \tau_{0} \cos n .
$$

In the same way, we can obtain

$$
\begin{aligned}
& g_{y}^{0}(s)=-b \tau_{0} \sin n-n, h_{x}^{0}(s)=b \tau_{0} \sin n+n, h_{y}^{0}(s)=a \tau_{0}-b \tau_{0} \cos n, \\
& g_{\varepsilon}^{0}(s)=0, g_{k}^{0}(s)=0, h_{\varepsilon}^{0}(s)=0, h_{k}^{0}(s)=0, g_{\varepsilon \varepsilon}^{0}(s)=0, g_{k k}^{0}(s)=0, g_{x x}^{0}(s)=0, \\
& g_{y y}^{0}(s)=0, h_{\varepsilon \varepsilon}^{0}(s)=0, h_{k k}^{0}(s)=0, h_{x x}^{0}(s)=0, h_{y y}^{0}(s)=0, g_{x \varepsilon}^{0}(s)=a-b \cos n, \\
& g_{y \varepsilon}^{0}(s)=-b \sin n, g_{x k}^{0}(s)=n b \tau_{0} \sin n, g_{y k}^{0}(s)=-n-b \tau_{0} \cos n, h_{x \varepsilon}^{0}(s)=b \sin n, \\
& h_{y \varepsilon}^{0}(s)=a-b \cos n, h_{x k}^{0}(s)=n+b \tau_{0} n \cos n, h_{y k}^{0}(s)=b \tau_{0} n \sin n, g_{x x y}^{0}(s)=0, \\
& h_{x x y}^{0}(s)=-\frac{\tau_{0}}{4}, g_{x y y}^{0}(s)=-\frac{\tau_{0}}{4}, h_{x y y}^{0}(s)=0, g_{x x \varepsilon}^{0}(s)=0, h_{x x \varepsilon}^{0}(s)=0, g_{x x k}^{0}(s)=0, \\
& h_{x x k}^{0}(s)=0, g_{y y \varepsilon}^{0}(s)=0, h_{y y \varepsilon}^{0}(s)=0, g_{y y k}^{0}(s)=0, h_{y y k}^{0}(s)=0, g_{x y \varepsilon}^{0}(s)=0, \\
& h_{x y \varepsilon}^{0}(s)=0, g_{x y k}^{0}(s)=0, h_{x y k}^{0}(s)=0 .
\end{aligned}
$$

By Taylor expansion, we know

$$
g(x, y, \varepsilon, k) \doteq\left[H+J k+L \varepsilon+N x^{2}+N y^{2}\right] x+[I+P k+M \varepsilon] y,
$$

$h(x, y, \varepsilon, k) \doteq[I+P k+M \varepsilon](-x)+\left[H+J k+L \varepsilon+N x^{2}+N y^{2}\right] y$, 
where

$$
\begin{gathered}
H=a \tau_{0}-b \tau_{0} \cos n, J=n b \tau_{0} \sin n, L=a-b \cos n, N=-\frac{1}{8} \tau_{0}, \\
I=-b \tau_{0} \sin n-n, P=-n-b \tau_{0} n \cos n, M=-b \sin n .
\end{gathered}
$$

From what has been discussed above, we have

Theorem 3.1. For system (1.1), near the Hopf bifurcation point $\tau_{0}$,

there exist periodic solutions of which the period is $\left(\tau_{0}+\varepsilon\right) \frac{2 \pi}{n}$, and the approximate analytic expression is

$$
\begin{aligned}
\bar{x}(s)= & x \sin n s+y \cos n s+\left(c_{1} \sin 3 n s+c_{2} \cos 3 n s\right) \frac{x^{3}}{6} \\
& +\left(-c_{2} \sin 3 n s+c_{1} \cos 3 n s\right) \frac{x^{2} y}{2}+\left(-c_{1} \sin 3 n s-c_{2} \cos 3 n s\right) \frac{x y^{2}}{2} \\
& +\left(c_{2} \sin 3 n s-c_{1} \cos 3 n s\right) \frac{y^{3}}{6}
\end{aligned}
$$

where

$$
\begin{array}{r}
s=s(t)=\frac{t}{\tau}(1+k), \quad \varepsilon \doteq \frac{P H-I J-P N\left(x^{2}+y^{2}\right)}{J M-P L}, \\
k \doteq \frac{L I-M H-M N\left(x^{2}+y^{2}\right)}{J M-P L} .
\end{array}
$$

\section{References}

[1] K. Engelborghs, T. Luzyanina, K. J. in $t$ Hout and D. Roose, Collocation methods for the computation of periodic solutions of delay differential equations, SIAM J. Sci. Comput. 22 (2000), 1593-1609.

[2] K. P. Hadeler, Effective computation of periodic orbits and bifurcation diagram in delay equations, Numer. Math. 34 (1980), 457-467.

[3] K. J. in $t$ Hout and Ch. Lubich, Periodic orbits of delay differential equations under discretization, BIT 38 (1998), 72-91.

[4] M. Martelli, Periodic solutions of some nonlinear delay-differential equations, J. Math. Anal. Appl. 74 (1980), 494-503. 
[5] H. I. Freedman and J. Wu, Periodic solutions of single species models with periodic delay, SIAM J. Math. Anal. 23 (1992), 689-701.

[6] T. Faria and L. T. Mahalháes, Normal forms for retarded functional differential equations with parameters and applications to Hopf bifurcation, J. Diff. Equation 122 (1995), 181-200.

[7] Z. Liu, P. Magal and S. Ruan, Hopf bifurcation for non-densely defined Cauchy problems, Zeitschrift fur Angewandte Mathematik und Physik 62 (2011), 191-222.

[8] S. J. Guo and J. H. Wu, Generalized Hopf bifurcation in delay differential equations (in Chinese), Sci. Sin. Math. 42 (2012), 91-105.

[9] Z. H. Yang and Q. Guo, Bifurcation analysis of delayed logistic equation, Applied Mathematics and Computation 167 (2005), 454-476.

[10] P. Chossat and M. Golubitsky, Hopf bifurcation in the presence of symmetry, center manifold and Liapunov-Schmidt reduction, Oscillations Bifurcation and Chaos 8 (1987), 343-352.

[11] S. L. Chang, C. S. Chien and B. W. Jeng, Liapunov-Schmidt reduction and continuation for nonlinear Schrödinger equations, SIAM Journal on Scientific Computing 29 (2007), 729-755.

[12] B. Temple and R. Young, A Liapunov-Schmidt reduction for time-periodic solutions of the compressible Euler equations, Methods and Applications of Analysis 17 (2010), $225-262$.

[13] B. Hassard, D. Kazarino and Y. Wan, Theory and Applications of Hopf Bifurcation, Cambridge University Press, Cambridge, 1981. 